\title{
Revisiting holographic superconductors with hyperscaling violation
}

\author{
Qiyuan Pan ${ }^{1,2,3, a}$, Shao-Jun Zhang ${ }^{1, b}$ \\ ${ }^{1}$ Instituto de Física, Universidade de São Paulo, C.P. 66318, 05315-970 São Paulo, Brazil \\ 2 Department of Physics, Key Laboratory of Low Dimensional Quantum Structures and Quantum Control of Ministry of Education, Hunan Normal \\ University, Changsha, Hunan 410081, China \\ ${ }^{3}$ State Key Laboratory of Theoretical Physics, Institute of Theoretical Physics, Chinese Academy of Sciences, Beijing 100190, China
}

Received: 3 December 2015 / Accepted: 18 February 2016 / Published online: 7 March 2016

(C) The Author(s) 2016. This article is published with open access at Springerlink.com

\begin{abstract}
We investigate the effect of the hyperscaling violation on the holographic superconductors. In the s-wave model, we find that the critical temperature decreases first and then increases as the hyperscaling violation increases, and the mass of the scalar field will not modify the value of the hyperscaling violation which gives the minimum critical temperature. We analytically confirm the numerical results by using the Sturm-Liouville method with the higher order trial function and improve the previous findings in Fan ( $\mathrm{J}$ High Energy Phys 09:048, 2013). However, different from the swave case, we note that the critical temperature decreases with the increase of the hyperscaling violation in the p-wave model. In addition, we observe that the hyperscaling violation affects the conductivity of the holographic superconductors and changes the expected relation in the gap frequency in both s-wave and p-wave models.
\end{abstract}

\section{Introduction}

The anti-de Sitter/conformal field theory (AdS/CFT) correspondence [1-4], which relates a $d$-dimensional quantum field theory with its dual gravitational theory, living in $(d+1)$ dimensions, has been employed to gain a better understanding of the high $T_{c}$ superconductor systems from the gravitational dual; for reviews, see Refs. [5-8] and references therein. It was suggested that the instability of the bulk black hole corresponds to a second order phase transition from normal state to superconducting state which brings about the spontaneous $U(1)$ symmetry breaking [9], and the properties of a $(2+1)$-dimensional superconductor can indeed be reproduced in the $(3+1)$-dimensional holographic dual model

\footnotetext{
a e-mail: panqiyuan@126.com

be-mail: sjzhang84@hotmail.com
}

where the AdS black hole geometry corresponds to a relativistic CFT at finite temperature $[10,11]$. Since many condensed matter systems do not have relativistic symmetry, it is of great interest to construct the corresponding holographic superconductor models by using the nonrelativistic version of the AdS/CFT correspondence. The holographic superconductors in the Lifshitz black hole spacetime were constructed in Refs. [12,13] and further investigated in Ref. [14]. It was observed that the Lifshitz black hole geometry results in different asymptotic behaviors of temporal and spatial components of gauge fields than those in the Schwarzschild-AdS black hole, which brings about some new features of holographic superconductor models [14]. Considering the holographic superconductors with Hořava-Lifshitz black holes, it was found that the holographic superconductivity is a robust phenomenon associated with asymptotic AdS black holes and the ratio of the gap frequency to the critical temperature is a little larger than the one in the relativistic situations [15]. Holographic superconductor models in the Lifshitz-like geometry can also be found, for example, in Refs. [16-26].

Recently, the so-called hyperscaling violation metric [2731], which can be considered as an extension of the Lifshitz metric, has received considerable attention due to its potential applications to the condensed matter physics [32-39]. Besides the anisotropic (Lifshitz) scaling is characterized by the dynamic critical exponent $z>1$ [40], the hyperscaling violation can also be used to describe holographically the realistic condensed matter systems with scaling properties going beyond the standard Lorentz scaling at criticality $[41,42]$. So it seems to be an interesting study to explore the effect of the hyperscaling violation on the holographic superconductors. More recently, the author of Ref. [43] introduced the holographic s-wave superconductors with hyperscaling violation and numerically found that the superconductivity still exists for the hyperscaling violation exponent 
$\theta=1$. Using the analytical Sturm-Liouville (S-L) method, which was first proposed in $[44,45]$ and later generalized to study holographic insulator/superconductor phase transition in $[46,47]$, it was found that the critical temperature increases as the hyperscaling violation increases [43]. However, using the shooting method $[10,11]$ to numerically study these holographic dual models in the full parameter space, we find that with the increase of the hyperscaling violation, the critical temperature decreases first and then increases. Obviously, the analytical result given in Ref. [43] misses some important information and does not agree well with our numerical calculation. Thus, we will reinvestigate the holographic s-wave superconductors with hyperscaling violation in this work. In addition to giving a more complete picture of how the hyperscaling violation affects the condensation for the scalar operator and the conductivity, we will present an interesting fact that the value of the hyperscaling violation, which gives the minimum critical temperature, remains unchanged even we vary the mass of the scalar field. Furthermore, compared with the second order trial function used in Ref. [43], we will improve the S-L method by including the higher order terms in the expansion of the trial function to reduce the disparity between the analytical and numerical results, and what's more, to obtain the analytical results which are completely consistent with the numerical findings.

Besides the holographic s-wave superconductor model, a holographic p-wave model can be realized by introducing a charged vector field in the bulk as a vector order parameter. The authors of Ref. [48] proposed a holographic p-wave model by adding an $S U$ (2) Yang-Mills field into the bulk, where a gauge boson generated by one $S U$ (2) generator is dual to the vector order parameter. Recently, Ref. [49] presented a new holographic p-wave superconductor model by introducing a charged vector field into an Einstein-Maxwell theory with a negative cosmological constant. In the probe limit, the black hole solution with non-trivial vector field can describe a superconducting phase and the ratio of the gap frequency to the critical temperature is given by $\omega_{g} / T_{c} \approx 8$, which is consistent with the s-wave model [50]. When taking the backreaction into account, a rich phase structure: zeroth order, first order and second order phase transitions has been observed in this p-wave model [51-53]. Considering a fivedimensional AdS soliton background coupled to a Maxwell complex vector field, in Ref. [54] the authors reconstructed the holographic p-wave insulator/superconductor phase transition model in the probe limit and showed that the EinsteinMaxwell complex vector field model is a generalization of the $S U$ (2) model with a general mass and gyromagnetic ratio. Other generalized investigations based on this new p-wave model can be found, for example, in Refs. [55-61]. Considering the increasing interest in study of the holographic p-wave model, we will also extend the study to the holographic pwave superconductor with hyperscaling violation, which has not been constructed as far as we know. We will observe that the hyperscaling violation has a completely different effect on the phase transitions for the holographic s-wave and p-wave superconductors, and the Maxwell complex vector model is still a generalization of the $S U(2)$ model even in the hyperscaling violation geometry. For simplicity and clarity, in this work we will concentrate on the probe limit where the backreaction of matter fields on the spacetime metric is neglected.

The plan of the work is the following. In Sect. 2 we will briefly review the black hole background with hyperscaling violation. In Sect. 3 we will explore the effect of the hyperscaling violation on the holographic s-wave superconductors. In Sect. 4 we will discuss the p-wave cases. We will conclude in the last section with our main results. We will derive, in Appendix A, the equations of motion for the $S U(2) \mathrm{p}$-wave superconductors with hyperscaling violation.

\section{Black hole solution with hyperscaling violation}

In order to study the effect of hyperscaling violation on the holographic superconductors in the probe limit, we consider the black hole solution with hyperscaling violation $[29,30]$

$\mathrm{d} s_{d+2}^{2}=r^{-2(d-\theta) / d}\left[-r^{-2(z-1)} f(r) \mathrm{d} t^{2}+\frac{\mathrm{d} r^{2}}{f(r)}+\mathrm{d} x_{i}^{2}\right]$,

with

$f(r)=1-\left(\frac{r}{r_{+}}\right)^{d+z-\theta}$,

where $\theta$ is the hyperscaling violation exponent, $z$ is the dynamical exponent and $r_{+}$is the radius of the event horizon. Obviously, at the asymptotic boundary $(r \rightarrow 0)$, we have

$\mathrm{d} s_{d+2}^{2}=r^{-2(d-\theta) / d}\left[-r^{-2(z-1)} \mathrm{d} t^{2}+\mathrm{d} r^{2}+\mathrm{d} x_{i}^{2}\right]$,

which is the most general metric that is spatially homogeneous and covariant under the scale transformations

$t \rightarrow \alpha^{z} t, \quad\left(r, x_{i}\right) \rightarrow \alpha\left(r, x_{i}\right), \quad \mathrm{d} s \rightarrow \alpha^{\theta / d} \mathrm{~d} s$,

with a real positive number $\alpha$. The novel feature of this metric is that the proper distance $\mathrm{d} s$ of the spacetime transforms non-trivially under scale transformations with the exponent $\theta$, which indicates a hyperscaling violation in the dual field theory $[29,30]$. Note that the Hawking temperature of the black hole is determined by

$T=\frac{d+z-\theta}{4 \pi r_{+}^{z}}$,

we can find that the thermal entropy, which is proportional to the area of the black hole, becomes [29]

$S \sim T^{(d-\theta) / z}$, 
which establishes that $\theta$ is the hyperscaling violation exponent. It should be noted that the metric (1) will reduce to the pure Lifshitz case when $\theta=0$ and $z \neq 1$, while it will describe the pure AdS case when $\theta=0$ and $z=1$.

For convenience in the following discussion, we introduce the coordinate transformation $u=r / r_{+}$and rewrite the metric (1) into

$$
\begin{aligned}
\mathrm{d} s_{d+2}^{2}= & \left(r_{+} u\right)^{-2(d-\theta) / d} \\
& \times\left[-\left(r_{+} u\right)^{-2(z-1)} f(u) \mathrm{d} t^{2}+\frac{r_{+}^{2}}{f(u)} \mathrm{d} u^{2}+\mathrm{d} x_{i}^{2}\right],
\end{aligned}
$$

with $f(u)=1-u^{d+z-\theta}$. Considering the validity of the solution and the requirement of the null energy condition, we get the constraint $[29,30]$

$d>\theta \geq 0, \quad z \geq 1+\frac{\theta}{d}$. $\psi^{\prime \prime}+\left(\frac{f^{\prime}}{f}-\frac{3-\theta}{u}\right) \psi^{\prime}+\left(\frac{r_{+}^{4} u^{2} \phi^{2}}{f^{2}}-\frac{m^{2} r_{+}^{\theta}}{u^{2-\theta} f}\right) \psi=0$

$\phi^{\prime \prime}+\frac{1}{u} \phi^{\prime}-\frac{2 r_{+}^{\theta} \psi^{2}}{u^{2-\theta} f} \phi=0$,

where the prime denotes the derivative with respect to $u$. Obviously, Eqs. (10) and (11) reduce to the ones in the standard holographic s-wave superconductors with $z=2$ Lifshitz scaling discussed in [14] when $\theta \rightarrow 0$. For completeness, we will also present the results for the case $\theta=0$ in the following.

In order to get the solutions in the superconducting phase, we have to count on the appropriate boundary conditions for $\psi$ and $\phi$. At the event horizon $u=1$ of the black hole, the regularity gives the boundary conditions

$\psi^{\prime}(1)=-\frac{m^{2} r_{+}^{\theta}}{4-\theta} \psi(1), \quad \phi(1)=0$.

At the asymptotic boundary $u \rightarrow 0$, the solutions behave like

$\psi= \begin{cases}\psi_{4-\Delta} r_{+}^{4-\Delta} u^{4-\Delta}+\psi_{\Delta} r_{+}^{\Delta} u^{\Delta}, & \text { with } \Delta=2+\sqrt{4+m^{2}} \text { for } \theta=0, \\ \psi_{0}+\psi_{\Delta} r_{+}^{\Delta} u^{\Delta}, & \text { with } \Delta=4-\theta \text { for } 0<\theta<2,\end{cases}$

In this work, we will set $d=2, z=2$ since we concentrate on the effect of hyperscaling violation $\theta$ on the holographic superconductors and compare with the results given in Ref. [43].

\section{S-wave superconductor models with hyperscaling violation}

In Ref. [43], the author constructed the holographic s-wave superconductors with hyperscaling violation and found that the critical temperature increases as the hyperscaling violation increases for the case of $z=2$ Lifshitz scaling. Now we will reinvestigate the effect of the hyperscaling violation on the holographic s-wave superconductors.

\subsection{Condensation and phase transition}

In the background of the four-dimensional hyperscaling violation black hole, we consider a gauge field and a scalar field coupled via the action

$S=\int \mathrm{d}^{4} x \sqrt{-g}\left(-\frac{1}{4} F_{\mu \nu} F^{\mu \nu}-|\nabla \psi-i A \psi|^{2}-m^{2}|\psi|^{2}\right)$.

Taking the ansatz of the matter fields as $\psi=\psi(u)$ and $A=\phi(u) \mathrm{d} t$, we can obtain the equations of motion from the action (9) for the scalar field $\psi$ and gauge field $\phi$, $\phi=\rho+\mu \ln u$.

According to the gauge/gravity duality, $\Delta$ is the conformal dimension of the scalar operator $\psi_{\Delta}=\langle O\rangle$ dual to the bulk scalar field, $\mu$ and $\rho$ are interpreted as the chemical potential and charge density in the dual field theory, respectively. Just as in Refs. [14,43], we will impose boundary condition $\psi_{4-\Delta}=0$ and $\psi_{0}=0$ since we focus on the condensate for the operator $\langle O\rangle$.

\subsubsection{Numerical investigation}

Using the shooting method $[10,11]$, we can solve numerically the equations of motion (10) and (11) by doing an integration from the horizon out to the boundary even if the hyperscaling violation exponent $\theta$ is fractional. Interestingly, from Eqs. (10) and (11) we can get the useful scaling symmetry and induced transformation of the relevant quantities,

$$
\begin{aligned}
& \psi \rightarrow \alpha^{-\theta / 2} \psi, \quad \phi \rightarrow \alpha^{-2} \phi \\
& \psi_{\Delta} \rightarrow \alpha^{-\left(\Delta+\frac{\theta}{2}\right)} \psi_{\Delta}, \quad \mu \rightarrow \alpha^{-2} \mu,
\end{aligned}
$$

which can be used to build the invariant and dimensionless quantities in the following calculation.

Changing the hyperscaling violation $\theta$, we present in Fig. 1 the condensate of the scalar operator $\langle O\rangle$ as a function of temperature for the fixed masses of the scalar field $m^{2} r_{+}^{\theta}=0$ (left) and $m^{2} r_{+}^{\theta}=-3$ (right). It is found that, for 


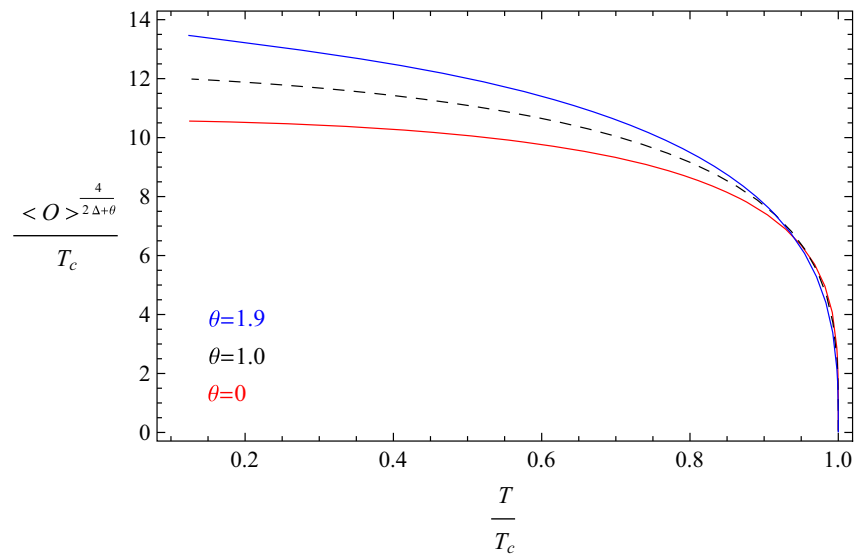

Fig. 1 The condensate of the scalar operator $\langle O\rangle$ as a function of temperature for the fixed masses of the scalar field $m^{2} r_{+}^{\theta}=0$ (left) and $m^{2} r_{+}^{\theta}=-3$ (right). In each panel, the three lines correspond to differ-

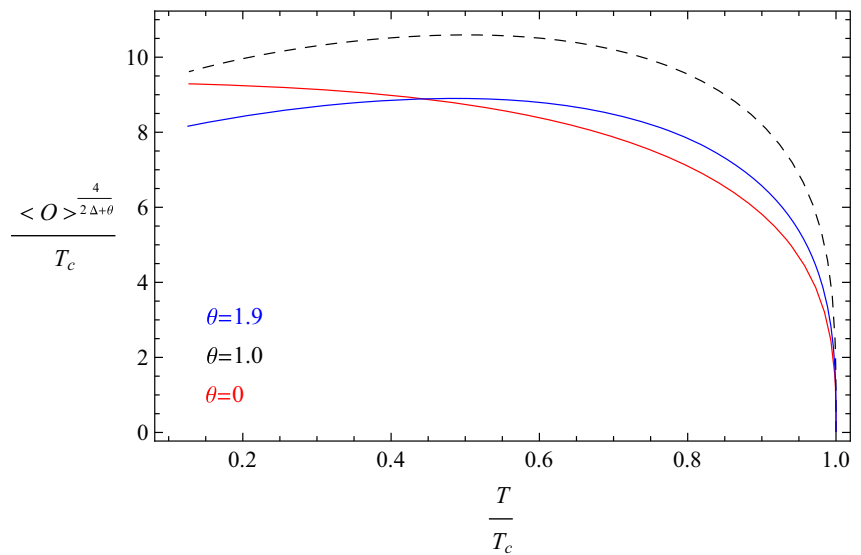

ent values of the hyperscaling violation, i.e., $\theta=0$ (red), 1.0 (black, and dashed) and 1.9 (blue), respectively

esting to note that the mass of the scalar field will not modify the value of the hyperscaling violation $\theta_{*} \approx 0.6$, which gives the minimum critical temperature $\theta$ and chosen various values of the hyperscaling violation $\mathrm{s}$-wave superconductor models with hyperscaling violation. It is inter-

\begin{tabular}{llllllll}
\hline$\theta$ & 0 & 0.5 & 0.6 & 0.7 & 1.0 & 1.5 & 1.9 \\
\hline$m^{2} r_{+}^{\theta}=0$ & $0.0229931 \mu$ & $0.0229413 \mu$ & $0.0229385 \mu$ & $0.0229387 \mu$ & $0.0229604 \mu$ & $0.0230883 \mu$ & $0.0233101 \mu$ \\
$m^{2} r_{+}^{\theta}=-1$ & $0.0253991 \mu$ & $0.0251925 \mu$ & $0.0251835 \mu$ & $0.0251853 \mu$ & $0.0252606 \mu$ & $0.0256748 \mu$ & $0.0264006 \mu$ \\
$m^{2} r_{+}^{\theta}=-2$ & $0.0289432 \mu$ & $0.0283204 \mu$ & $0.0282962 \mu$ & $0.0283004 \mu$ & $0.0284872 \mu$ & $0.0295178 \mu$ & $0.0314512 \mu$ \\
$m^{2} r_{+}^{\theta}=-3$ & $0.0351935 \mu$ & $0.0330971 \mu$ & $0.0330311 \mu$ & $0.0330385 \mu$ & $0.0334913 \mu$ & $0.0361219 \mu$ & $0.0420384 \mu$ \\
\hline
\end{tabular}

all cases considered here, the scalar operator $\langle O\rangle$ is singlevalued near the critical temperature and the condensate drops to zero continuously at the critical temperature. By fitting these curves, we see that for small condensate there is a square root behavior,

$\langle O\rangle \sim\left(1-T / T_{c}\right)^{1 / 2}$

which is typical of second order phase transitions with the mean field critical exponent $1 / 2$ for all values of $\theta$. The behaviors of the condensate for the scalar operator $\langle O\rangle$ show that the holographic s-wave superconductors still exist even in the background of the hyperscaling violation black hole.

In order to obtain the effect of the hyperscaling violation on the critical temperature $T_{c}$, we give the critical temperature $T_{c}$ for the scalar operator $\langle O\rangle$ when we fix the masses of the scalar field $m^{2} r_{+}^{\theta}=0,-1,-2$, and -3 for different hyperscaling violation exponent $\theta$ in Table 1 . For the same mass of the scalar field, it is clear that with the increase of the hyperscaling violation $\theta$, the critical temperature $T_{c}$ decreases first and then increases, which is different from the result obtained in Ref. [43] where the critical temperature was shown to increase almost linearly when $\theta$ increases. Obviously, from Table 1, we find that there exists a mini- mum value of the critical temperature at $\theta_{*} \approx 0.6$ for the fixed mass of the scalar field, which implies that the higher hyperscaling violation makes it harder for the condensation to form in the range $0 \leq \theta<\theta_{*}$ but easier in the range $\theta_{*}<\theta<2$. Interestingly, we observe that the mass of the scalar field will not alter the value of $\theta_{*}$. It should be noted that how the hyperscaling violation works in the holographic s-wave superconductors is still an open question.

\subsubsection{Analytical understanding}

Using the S-L method $[44,45]$, Fan analytically explored the effect of the hyperscaling violation on the s-wave superconducting transition temperature and found that the critical temperature increases with the increase of hyperscaling violation [43]. Obviously, the analytical result given in Ref. [43] is not in agreement with our numerical calculation and some important information is missing. We will improve the S-L method to get an analytical result which is consistent with the numerical calculation.

At the critical temperature $T_{c}$, the scalar field $\psi=0$. Thus, near the critical point the equation of motion (11) for the gauge field $\phi$ reduces to 
$\phi^{\prime \prime}+\frac{1}{u} \phi^{\prime}=0$.

Considering the boundary condition (12) for $\phi$, we can get the solution to Eq. (17),

$\phi(u)=\lambda r_{+c}^{-2} \ln u$,

where we have set $\lambda=\mu r_{+c}^{2}$ with the radius of the horizon at the critical point $r_{+c}$.

Defining a trial function $F(u)$ which matches the boundary behavior (13) for $\psi$

$\psi(u) \sim\langle O\rangle r_{+}^{\Delta} u^{\Delta} F(u)$,

with the boundary conditions $F(0)=1$ and $F^{\prime}(0)=0$, from Eq. (10) we can obtain the equation of motion for $F(u)$

$\left(Q F^{\prime}\right)^{\prime}+Q\left(U+\lambda^{2} V\right) F=0$

with

$$
\begin{aligned}
& Q(u)=u^{2 \Delta+\theta-3} f, \\
& U(u)=\frac{\Delta f^{\prime}}{u f}+\frac{\Delta(\Delta+\theta-4)}{u^{2}}-\frac{m^{2} r_{+}^{\theta}}{u^{2-\theta} f}, \\
& V(u)=\left(\frac{u \ln u}{f}\right)^{2} .
\end{aligned}
$$

According to the S-L eigenvalue problem [62], we can deduce that the eigenvalue $\lambda$ minimizes the expression

$$
\begin{aligned}
\lambda^{2} & =\lambda_{a b}^{2} \\
& =\frac{0.5-0.785714 a+0.490385 a^{2}-0.983333 a b+0.705357 b+0.508824 b^{2}}{0.0137786-0.0147955 a+0.00459295 a^{2}-0.00750497 a b+0.0114973 b+0.00312254 b^{2}},
\end{aligned}
$$

$\lambda^{2}=\frac{\int_{0}^{1} Q\left(F^{\prime 2}-U F^{2}\right) \mathrm{d} u}{\int_{0}^{1} Q V F^{2} \mathrm{~d} u}$.

Using Eq. (22) to compute the minimum eigenvalue of $\lambda^{2}$, we can get the critical temperature $T_{c}$ for different hyperscaling violation $\theta$ and mass of the scalar field $m^{2} r_{+}^{\theta}$ from the following relation:

$T_{c}=\frac{4-\theta}{4 \pi \lambda_{\min }} \mu$.

Before going further, we would like to make a comment. Considering the boundary conditions of $F(u)$, i.e., $F(0)=1$ and $F^{\prime}(0)=0$, people usually assume the trial function to be

$F(u)=F_{a}(u)=1-a u^{2}$, with a constant $a$, just as done in Ref. [43]. It should be noted that this assumption works well in most cases $[44,45$, 63-76]. Unfortunately, using this trial function in our case, we find that the analytical results are not in agreement with the numerical calculation and some important information is missing. To solve this problem, we choose the trial function by including the higher order of $u$ such as the third order trial function

$F(u)=F_{a b}(u)=1-a u^{2}+b u^{3}$,

with two constants $a$ and $b$. We prefer (25) over (24) because it gives a better estimate of the minimum of (22), which means that the analytical results are much closer to the numerical findings, and more importantly, it can correctly reveal the influence of the hyperscaling violation on the critical temperature $T_{c}$.

As an example, we will calculate the case for the fixed mass of the scalar field $m^{2} r_{+}^{\theta}=-3$ with the chosen value of the hyperscaling violation $\theta=1.5$. If we choose the trial function (24), we have

$\lambda^{2}=\lambda_{a}^{2}=\frac{0.5-0.785714 a+0.490385 a^{2}}{0.0137786-0.0147955 a+0.00459295 a^{2}}$,

whose minimum is $\lambda^{2}=30.4899$ at $a=0.477530$. According to Eq. (22), we can easily obtain the critical temperature $T_{c}=0.036029 \mu$. Changing the trial function into the form (25), we arrive at whose minimum is $\lambda^{2}=30.3335$ at $a=0.913984$ and $b=0.403370$. Hence the critical temperature reads $T_{c}=$ $0.0361217 \mu$. Comparing with the analytical result from the trial function $F_{a}(u)$, we find that this value is much closer to the numerical result $T_{c}=0.0361219 \mu$ given in Table 1 and the difference between the analytical and numerical values is $0.0004 \%$ ! In Fig. 2, we plot the trial function $F_{a}(u)=$ $1-a u^{2}$ (green) and $F_{a b}(u)=1-a u^{2}+b u^{3}$ (red) as a function of $u$ when $\lambda^{2}$ attains its minimum for the fixed mass of the scalar field $m^{2} r_{+}^{\theta}=-3$ and hyperscaling violation $\theta=1.5$, which clearly shows the difference between these two trial functions.

Extending the analytical investigation to the holographic s-wave superconductors with hyperscaling violation in the full parameter space (8), we can get the critical temperature $T_{c}$ with various masses of the scalar field $m^{2} r_{+}^{\theta}$ and hyper- 


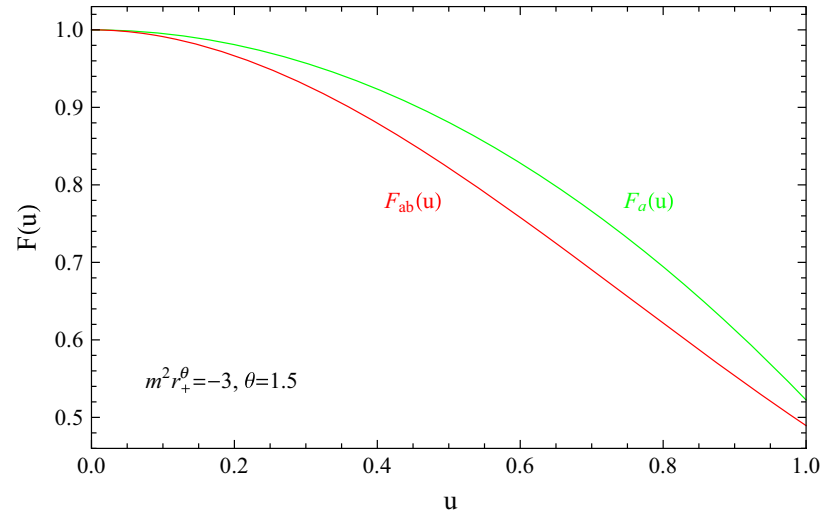

Fig. 2 The trial function $F_{a}(u)=1-a u^{2}$ (green) and $F_{a b}(u)=$ $1-a u^{2}+b u^{3}(r e d)$ as a function of $u$ when $\lambda^{2}$ attains its minimum for the fixed mass of the scalar field $m^{2} r_{+}^{\theta}=-3$ and hyperscaling violation $\theta=1.5$

scaling violation $\theta$ for the scalar operator $\langle O\rangle$. To see the dependence of the analytical results on the hyperscaling violation more directly and compare with Fig. 3 of Ref. [43], we exhibit the critical temperature $T_{c}$ as a function of the hyperscaling violation $\theta$ for the fixed masses of the scalar field $m^{2} r_{+}^{\theta}=0$ (left) and $m^{2} r_{+}^{\theta}=-3$ (right) in Fig. 3. We also present the numerical results obtained by using the shooting method in order to compare with the analytical results. Obviously, compared with the trial function $F_{a}(u)$, the third order trial function $F_{a b}(u)$ can indeed be used to further improve the analytical results and reduce the disparity between the analytical and numerical results. Furthermore, in contrast to the trial function $F_{a}(u)$, which only tells us that the critical temperature $T_{c}$ increases with the increase of hyperscaling violation $\theta$, the third order trial function $F_{a b}(u)$ can be used to give the analytical results which are completely consistent with the numerical findings. Thus, we conclude that we can still count on the S-L method with the higher order trial func-

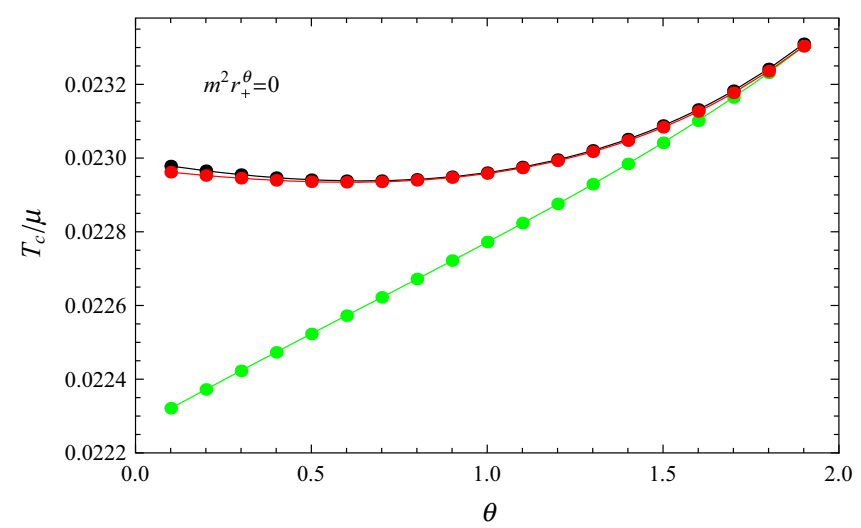

Fig. 3 The critical temperature $T_{c}$ as a function of the hyperscaling violation $\theta$ with fixed masses of the scalar field $m^{2} r_{+}^{\theta}=0$ (left) and $m^{2} r_{+}^{\theta}=-3$ (right) in the holographic s-wave superconductors with hyperscaling violation. The three lines from top to bottom correspond tion $F(u)$ to analytically study the effect of the hyperscaling violation on the holographic s-wave superconductors.

Now we are in a position to study the critical phenomena of the holographic s-wave superconductors with hyperscaling violation. Since the condensation for the scalar operator $\langle O\rangle$ is so small when $T \rightarrow T_{c}$, we can expand $\phi(u)$ in $\langle O\rangle$ as

$\phi(u)=\lambda r_{+}^{-2} \ln u+r_{+}^{-2} \mathcal{A} \chi(u)+\cdots$,

where we have introduced $\mathcal{A}=r_{+}^{2 \Delta+\theta}\langle O\rangle^{2}$ and the boundary conditions $\chi(1)=0$ and $\chi^{\prime}(1)=0$ [44,45]. Thus, substituting the functions (19) and (28) into (11), we can get the equation of motion for $\chi(u)$

$\left(u \chi^{\prime}\right)^{\prime}=\frac{2 \lambda u^{2 \Delta+\theta-1} F^{2} \ln u}{f}$.

Making integration of both sides of Eq. (29), we have

$\left.\left(u \chi^{\prime}\right)\right|_{u \rightarrow 0}=\lambda \mathcal{C}=-\lambda \int_{0}^{1} \frac{2 u^{2 \Delta+\theta-1} F^{2} \ln u}{f} \mathrm{~d} u$.

From Eqs. (18) and (28), near $u \rightarrow 0$ we can arrive at

$\mu=\lambda r_{+}^{-2}+\left.r_{+}^{-2} \mathcal{A}\left(u \chi^{\prime}\right)\right|_{u \rightarrow 0}$,

which leads to

$\langle O\rangle=\frac{1}{\sqrt{\mathcal{C}}}\left(\frac{4 \pi T_{c}}{4-\theta}\right)^{\frac{2 \Delta+\theta}{4}}\left(1-\frac{T}{T_{c}}\right)^{\frac{1}{2}}$.

Obviously, Eq. (32) is valid for all cases considered here. For example, for the case of $\theta=1.5$ with $m^{2} r_{+}^{\theta}=-3$, we have $\langle O\rangle=0.269906\left(1-T / T_{c}\right)^{1 / 2}$ when $a=0.913984$ and $b=0.403370$, which agrees well with the numerical calculation by using the shooting method. Especially, for the case of $\theta=0$ with $m^{2}=-3$, we obtain $\langle O\rangle=0.249154(1-$ $\left.T / T_{c}\right)^{1 / 2}$ when $a=1.64069$ and $b=0.911971$, which is in good agreement with the numerical result given in [14]. Since the hyperscaling violation and mass of the scalar field

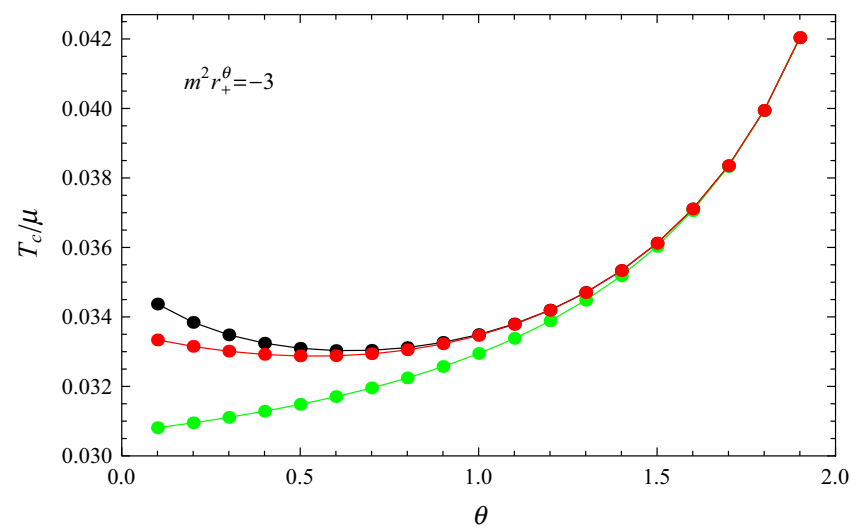

to the results obtained by the numerical calculation (black) and from the analytical S-L method by using the trial function $F(u)=1-a u^{2}+b u^{3}$ (red) and $F(u)=1-a u^{2}$ (green), respectively 

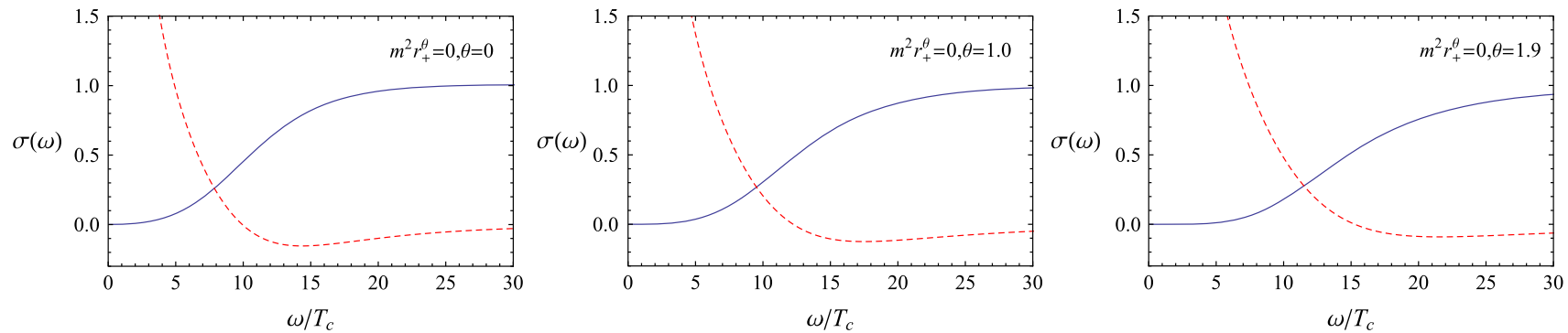
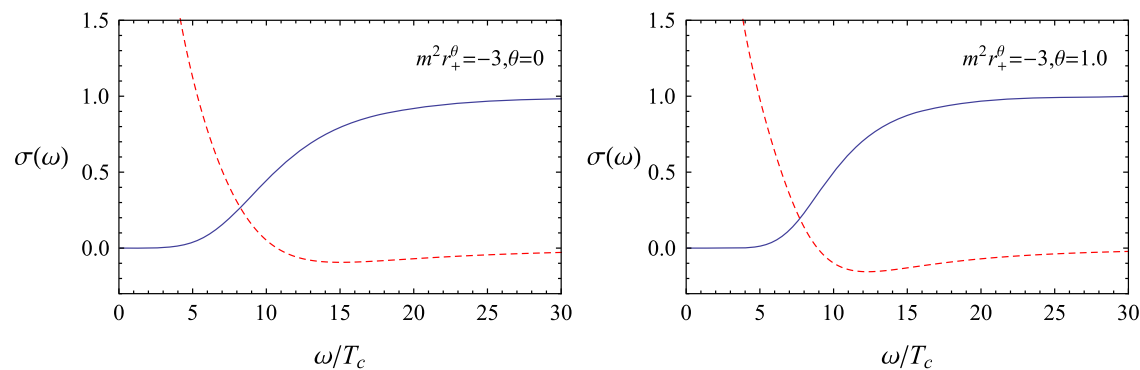

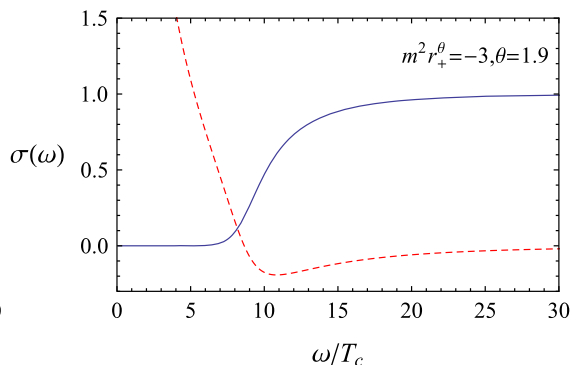

Fig. 4 Conductivity of the holographic s-wave superconductors with hyperscaling violation for the fixed masses of the scalar field and different values of the hyperscaling violation. In each panel, the blue (solid) line and the red (dashed) line represent the real part and imaginary part of the conductivity $\sigma(\omega)$, respectively will not alter Eq. (32) except for the prefactor, we can reproduce the expression (16) near the critical point by using the analytical S-L method. It shows that the holographic s-wave superconducting phase transition with hyperscaling violation is of the second order and the critical exponent of the system always takes the mean-field value $1 / 2$. The hyperscaling violation will not influence the result.

\subsection{Conductivity}

In [43], it was found that a gap opens in the real part of the conductivity in the holographic s-wave superconductor models with hyperscaling violation, which indicates the onset of superconductivity. Considering that the author only concentrated on the case of the hyperscaling violation $\theta=1.0$, we will vary $\theta$ to discuss the effect of the hyperscaling violation on the conductivity.

Assuming that the perturbed Maxwell field has a form $\delta A_{x}=A_{x}(u) e^{-i \omega t} \mathrm{~d} x$, we obtain the equation of motion for $A_{x}$, which can be used to calculate the conductivity

$A_{x}^{\prime \prime}+\left(\frac{f^{\prime}}{f}-\frac{1}{u}\right) A_{x}^{\prime}+\left(\frac{r_{+}^{4} \omega^{2} u^{2}}{f^{2}}-\frac{2 r_{+}^{\theta} \psi^{2}}{u^{2-\theta} f}\right) A_{x}=0$.

For different hyperscaling violation exponents, the ingoing wave boundary condition near the horizon is given by

$A_{x}(u) \sim(1-u)^{-\frac{i \omega}{4 \pi T}}$ and the general behavior in the asymptotic region $(u \rightarrow 0)$ can be written as

$A_{x}=A_{x}^{(0)}+A_{x}^{(1)} r_{+}^{2} u^{2}$.

Thus, we can obtain the conductivity of the dual superconductor by using the gauge/gravity duality [10,11,43]

$\sigma=-\frac{2 i A_{x}^{(1)}}{\omega A_{x}^{(0)}}$.

For different values of the hyperscaling violation $\theta$, one can obtain the conductivity by solving the Maxwell equation numerically. We still focus on the case for the fixed scalar masses $m^{2} r_{+}^{\theta}=0$ and $m^{2} r_{+}^{\theta}=-3$ in our discussion.

In Fig. 4 we plot the frequency dependent conductivity of the holographic s-wave superconductors with hyperscaling violation by solving the Maxwell equation (33) numerically for $\theta=0,1.0$, and 1.9 with $m^{2} r_{+}^{\theta}=0$ and $m^{2} r_{+}^{\theta}=-3$ at temperatures $T / T_{c} \approx 0.15$. In each panel, the blue (solid) line and red (dashed) line represent the real part and imaginary part of the conductivity $\sigma(\omega)$, respectively. For all cases considered here, we find a gap in the conductivity which is parameterized by the gap frequency $\omega_{g}$. Defining $\omega_{g}$ by the minimum of $|\sigma|$ [50], we observe that with the increase of the hyperscaling violation $\theta$, the gap frequency $\omega_{g}$ becomes larger. Also, for increasing hyperscaling violation, we have larger deviation from the value $\omega_{g} / T_{c} \approx 8$, especially for the case of $m^{2} r_{+}^{\theta}=0$. This shows that the hyperscaling violation really affects the conductivity of the holographic s-wave superconductors and change the ratio in the gap frequency $\omega_{g} / T_{c} \approx 8$, which was claimed to be universal in [50]. 


\section{P-wave superconductor models with hyperscaling violation}

Since the hyperscaling violation has the interesting effect on the holographic s-wave superconductors, which greatly improves the previous findings in Ref. [43], it seems worthwhile to consider the influence of the hyperscaling violation on the holographic p-wave superconductors which has not been constructed as far as we know.

\subsection{Condensation and phase transition}

Working in the probe limit, we will construct the p-wave holographic superconductor models with hyperscaling violation via the Maxwell complex vector field model $[49,51]$ of equations of motion are the same if we set $m^{2} r_{+}^{\theta}=0$ and rescale the field by $\rho_{x}(u)=\psi(u) / \sqrt{2}$ in Eqs. (39) and (40). Thus, in this sense, the complex vector field model is still a generalization of the $S U$ (2) Yang-Mills model in the holographic superconductors with hyperscaling violation, which supports the argument given in [54].

We will impose the appropriate boundary conditions for $\rho_{x}(u)$ and $\phi(u)$ to get the solutions in the superconducting phase. Interestingly, we observe that $\phi$ has the same boundary conditions just as in Eq. (12) for the horizon $u=1$ and Eq. (14) for the boundary $u \rightarrow 0$. But for the vector field $\rho_{x}$, we find at the horizon

$\rho_{x}^{\prime}(1)=-\frac{m^{2} r_{+}^{\theta}}{4-\theta} \rho_{x}(1)$,

and at the asymptotic boundary

$\rho_{x}= \begin{cases}\rho_{x}^{(2-\Delta)} r_{+}^{2-\Delta} u^{2-\Delta}+\rho_{x}^{(\Delta)} r_{+}^{\Delta} u^{\Delta}, & \text { with } \Delta=1+\sqrt{1+m^{2}} \text { for } \theta=0, \\ \rho_{x}^{(0)}+\rho_{x}^{(\Delta)} r_{+}^{\Delta} u^{\Delta}, & \text { with } \Delta=2 \text { for } 0<\theta<2,\end{cases}$

$$
\begin{aligned}
S=\int & \mathrm{d}^{4} x \sqrt{-g} \times\left(-\frac{1}{4} F_{\mu \nu} F^{\mu \nu}-\frac{1}{2} \rho_{\mu \nu}^{\dagger} \rho^{\mu \nu}\right. \\
& \left.-m^{2} \rho_{\mu}^{\dagger} \rho^{\mu}+i q \gamma \rho_{\mu} \rho_{\nu}^{\dagger} F^{\mu \nu}\right),
\end{aligned}
$$

where the strength of $U(1)$ field $A_{\mu}$ is $F_{\mu \nu}=\nabla_{\mu} A_{\nu}-\nabla_{\nu} A_{\mu}$ and the tensor $\rho_{\mu \nu}$ is defined by $\rho_{\mu \nu}=D_{\mu} \rho_{\nu}-D_{\nu} \rho_{\mu}$ with the covariant derivative $D_{\mu}=\nabla_{\mu}-i q A_{\mu} . m$ and $q$ represent the mass and charge of the vector field $\rho_{\mu}$, respectively. Since we will consider the case without external magnetic field, the parameter $\gamma$, which describes the interaction between the vector field $\rho_{\mu}$ and gauge field $A_{\mu}$, will not play any role.

Just as in Refs. [49,51], we will adopt the following ansatz for the matter fields:

$\rho_{\nu} \mathrm{d} x^{\nu}=\rho_{x}(u) \mathrm{d} x, \quad A_{\nu} \mathrm{d} x^{\nu}=\phi(u) \mathrm{d} t$,

where we can set $\rho_{x}$ to be real by using the $U(1)$ gauge symmetry. Thus, from the solution (7) for $d=2, z=2$ and action (37), we can get the equations of motion for the vector hair $\rho_{x}$ and gauge field $\phi$

$\rho_{x}^{\prime \prime}+\left(\frac{f^{\prime}}{f}-\frac{1}{u}\right) \rho_{x}^{\prime}+\left(\frac{r_{+}^{4} u^{2} \phi^{2}}{f^{2}}-\frac{m^{2} r_{+}^{\theta}}{u^{2-\theta} f}\right) \rho_{x}=0$,

$\phi^{\prime \prime}+\frac{1}{u} \phi^{\prime}-\frac{2 r_{+}^{2} \rho_{x}^{2}}{f} \phi=0$,

where the prime denotes the derivative with respect to $u$. Without loss of generality, we have scaled $q=1$ as in Ref. [49]. Comparing the above two equations of motion with Eqs. (A3) and (A4) for the holographic p-wave superconductors with hyperscaling violation in the $S U$ (2) Yang-Mills system constructed in the appendix, we can find that the two sets where $\rho_{x}^{(2-\Delta)}$ (or $\left.\rho_{x}^{(0)}\right)$ and $\rho_{x}^{(\Delta)}$ are interpreted as the source and vacuum expectation value of the vector operator $J_{x}$ in the dual field theory according to the gauge/gravity duality, respectively. In this work, we will impose the boundary condition $\rho_{x}^{(2-\Delta)}=0$ (or $\rho_{x}^{(0)}=0$ ) since we require that the condensate appears spontaneously.

We will count on the shooting method $[10,11]$ to solve numerically the equations of motion (39) and (40) in this section. In the following calculation, we will use the scaling symmetry from the equations of motion and induced transformation of the relevant quantities, i.e.,

$$
\begin{aligned}
\rho_{x} & \rightarrow \alpha^{-1} \rho_{x}, \phi \rightarrow \alpha^{-2} \phi, \\
\rho_{x}^{(\Delta)} & \rightarrow \alpha^{-(1+\Delta)} \rho_{x}^{(\Delta)}, \quad \mu \rightarrow \alpha^{-2} \mu,
\end{aligned}
$$

to build the invariant and dimensionless quantities.

In Fig. 5, we present the condensate of the vector operator $\left\langle J_{x}\right\rangle$ as a function of temperature for different hyperscaling violation exponents $\theta$ with fixed masses of the vector field $m^{2} r_{+}^{\theta}=0$ (left) and $m^{2} r_{+}^{\theta}=5 / 4$ (right) in the holographic pwave superconductor model. Obviously, the behavior of each curve for the fixed $\theta$ and $m^{2} r_{+}^{\theta}$ agrees well with the holographic superconducting phase transition in the literature, which shows that the black hole solution with a non-trivial vector field can describe a superconducting phase. Similar to the s-wave case in Fig. 1, it is observed that, for all cases considered here, the vector operator $\left\langle J_{x}\right\rangle$ is single-valued near the critical temperature and the condensate drops to zero continuously at the critical temperature. Fitting these curves, we obtain a square root behavior for the small condensate

$\left\langle J_{x}\right\rangle \sim\left(1-T / T_{c}\right)^{1 / 2}$, 


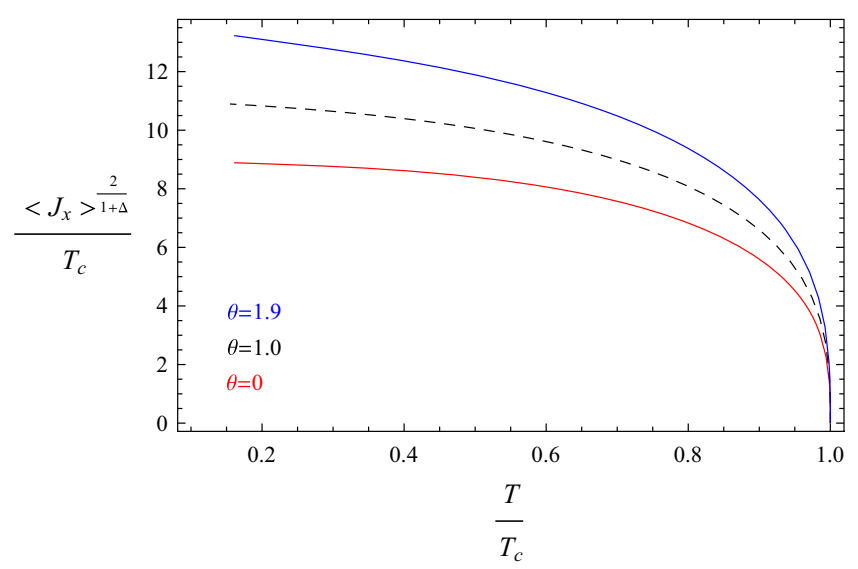

Fig. 5 The condensate of the vector operator $\left\langle J_{x}\right\rangle=\rho_{x}^{(\Delta)}$ as a function of temperature for the fixed masses of the vector field $m^{2} r_{+}^{\theta}=0$ (left) and $m^{2} r_{+}^{\theta}=5 / 4$ (right). In each panel, the three lines from bottom to

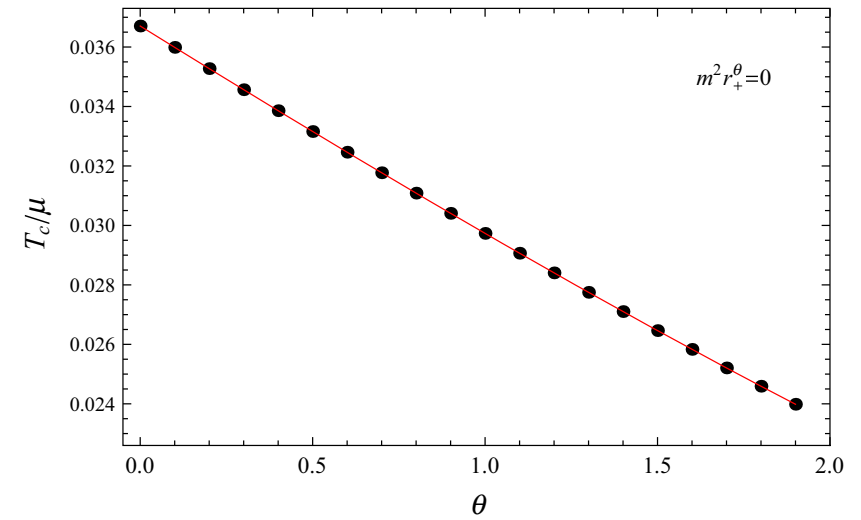

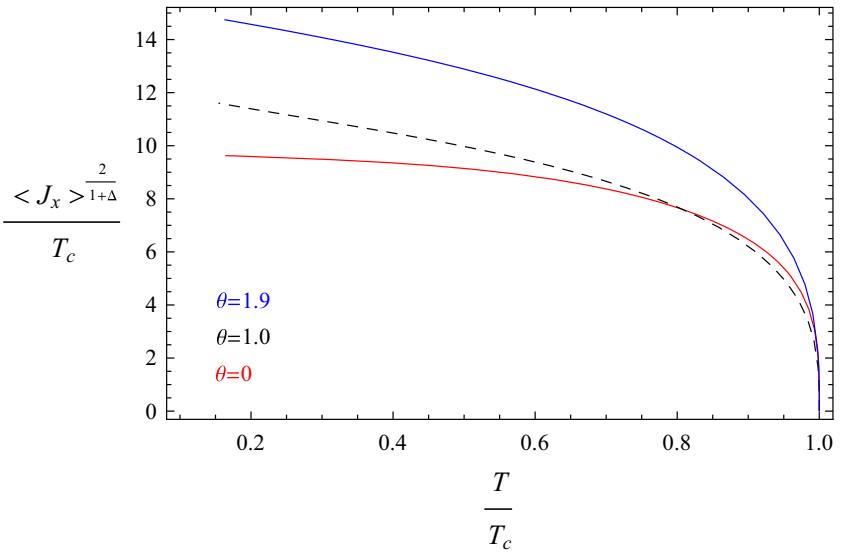

top correspond to increasing hyperscaling violation, i.e., $\theta=0$ (red), 1.0 (black and dashed), and 1.9 (blue), respectively

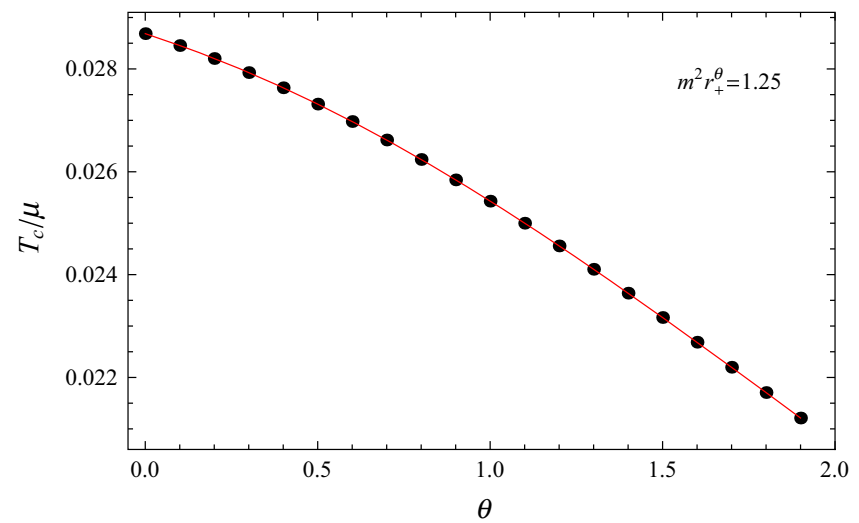

Fig. 6 The critical temperature $T_{c}$ obtained by the numerical method as a function of the hyperscaling violation $\theta$ with fixed masses of the vector field $m^{2} r_{+}^{\theta}=0(l e f t)$ and $m^{2} r_{+}^{\theta}=5 / 4(r i g h t)$ in the holographic p-wave superconductors with hyperscaling violation

which means that the phase transition belongs to the second order and the critical exponent of the system takes the meanfield value $1 / 2$.

In order to show the effect of the hyperscaling violation on the critical temperature $T_{c}$ in the holographic pwave superconductors, in Fig. 6 we plot the critical temperature $T_{c}$ as a function of the hyperscaling violation $\theta$ with fixed masses of the vector field $m^{2} r_{+}^{\theta}=0$ (left) and $m^{2} r_{+}^{\theta}=5 / 4$ (right). We find that the critical temperature $T_{c}$ for the vector operator $\left\langle J_{x}\right\rangle$ with the fixed vector field mass decreases as the hyperscaling violation $\theta$ increases, which implies that the higher hyperscaling violation makes it harder for the condensation to form in the full parameter space (8). Obviously, this behavior is different from that seen for the holographic s-wave superconductors with hyperscaling violation in Fig. 3, where the critical temperature $T_{c}$ decreases first and then increases as the hyperscaling violation increases. Thus, we argue that, although the underlying mechanism remains mysterious, the hyperscaling violation has completely different effect on the critical tem- perature for the s-wave and p-wave superconductor phase transitions.

\subsection{Conductivity}

In order to calculate the conductivity, we will consistently turn on the perturbation of $A_{y}$ only and assume the form of the perturbed Maxwell field $\delta A_{y}=A_{y}(u) e^{-i \omega t} \mathrm{~d} y$, which leads to the equation of motion

$$
A_{y}^{\prime \prime}+\left(\frac{f^{\prime}}{f}-\frac{1}{u}\right) A_{y}^{\prime}+\left(\frac{r_{+}^{4} \omega^{2} u^{2}}{f^{2}}-\frac{2 r_{+}^{2} \rho_{x}^{2}}{f}\right) A_{y}=0
$$

Considering the ingoing wave boundary condition near the horizon

$A_{y}(u) \sim(1-u)^{-\frac{i \omega}{4 \pi T}}$

and the behavior in the asymptotic region $(u \rightarrow 0)$

$A_{y}=A_{y}^{(0)}+A_{y}^{(1)} r_{+}^{2} u^{2}$, 

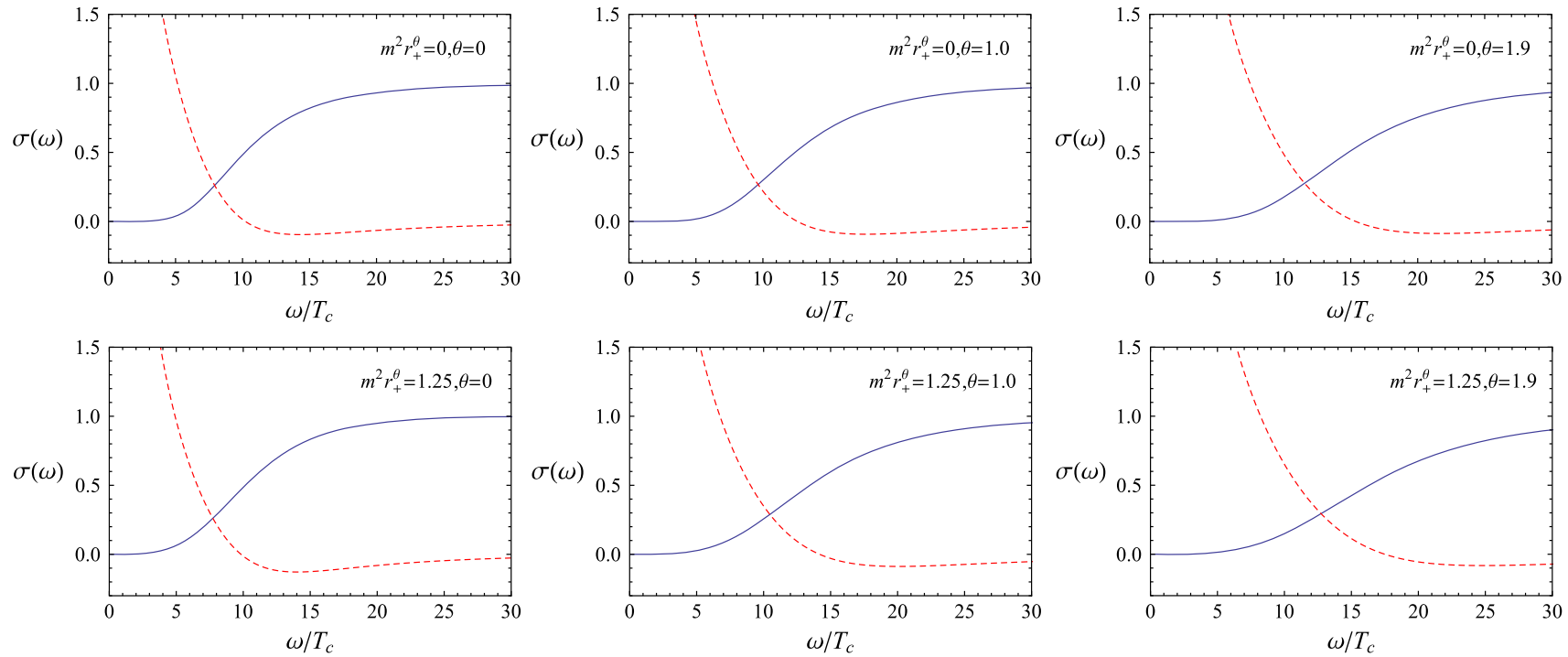

Fig. 7 Conductivity of the holographic p-wave superconductors with hyperscaling violation for the fixed masses of the vector field and different values of the hyperscaling violation. In each panel, the blue (solid)

line and red (dashed) line represent the real part and imaginary part of the conductivity $\sigma(\omega)$, respectively

we can express the conductivity as $[10,11]$

$\sigma=-\frac{2 i A_{y}^{(1)}}{\omega A_{y}^{(0)}}$.

We still choose the masses of the vector field $m^{2} r_{+}^{\theta}=0$ and $m^{2} r_{+}^{\theta}=5 / 4$ in our calculation.

Solving the Maxwell equation (45) numerically for $\theta=0$, 1.0 , and 1.9 with $m^{2} r_{+}^{\theta}=0$ and $m^{2} r_{+}^{\theta}=5 / 4$ at temperatures $T / T_{C} \approx 0.15$, we present the frequency dependent conductivity of the holographic p-wave superconductors with hyperscaling violation in Fig. 7. Similar to the s-wave case in Fig. 4, for all cases considered here, we observe that the conductivity develops a gap with the gap frequency $\omega_{g}$ and the larger deviation from the value $\omega_{g} / T_{c} \approx 8$ as the hyperscaling violation $\theta$ increases. Therefore, we conclude that the higher hyperscaling violation results in the larger deviation from the universal value $\omega_{g} / T_{c} \approx 8$ [50] for the gap frequency in both s-wave and p-wave holographic superconductor models.

\section{Conclusions}

We have investigated the holographic superconductors with hyperscaling violation in the probe limit, which may help to understand the condensed matter materials with scaling properties going beyond the standard Lorentz scaling at criticality. In the s-wave (scalar field) model, different from the findings as shown in Ref. [43] that the critical temperature increases as the hyperscaling violation increases for the case of $z=2$ Lifshitz scaling, we found that the critical temperature decreases first and then increases with the increase of the hyperscaling violation, which implies that the increase of the hyperscaling violation makes the condensation of the scalar operator harder for small $\theta$ but easier for large $\theta$. Interestingly, the mass of the scalar field will not modify the value of the hyperscaling violation $\theta_{*} \approx 0.6$, which gives the minimum critical temperature. We improved the S-L method by including the higher order terms in the expansion of the trial function to confirm our numerical results and argued that, compared with the second order trial function used in Ref. [43], the higher order trial function can indeed be used to give the analytical results which are completely consistent with the numerical findings. However, the story is completely different if we extend the investigation to the $\mathrm{p}$-wave (vector field) model. In contrast to the s-wave model, we observed in the p-wave case that the critical temperature decreases as the hyperscaling violation increases, which tells us that the higher hyperscaling violation makes it harder for the vector condensation to form in the full parameter space. Thus, we concluded that, although the underlying mechanism remains mysterious, the hyperscaling violation has a completely different effect on the phase transitions for the holographic s-wave and p-wave superconductors. On the other hand, it should be noted that the Maxwell complex vector model is still a generalization of the $S U$ (2) model even in the hyperscaling violation geometry. Moreover, we pointed out that the hyperscaling violation affects the conductivity of the holographic superconductors and the higher hyperscaling violation results in the larger deviation from the universal value $\omega_{g} / T_{c} \approx 8$ for the gap frequency in both s-wave and p-wave models. The extension of this work to the fully backreacted spacetime would be 
interesting since the backreaction provides richer physics in the holographic superconductor models. We will leave this for further study.

Acknowledgments We thank Professor Elcio Abdalla for his helpful discussions and suggestions. This work was supported by FAPESP No. 2013/26173-9 and CNPq (Brazil); the National Natural Science Foundation of China under Grant No. 11275066; and the Open Project Program of State Key Laboratory of Theoretical Physics, Institute of Theoretical Physics, Chinese Academy of Sciences, China (No. Y5KF161CJ1).

Open Access This article is distributed under the terms of the Creative Commons Attribution 4.0 International License (http://creativecomm ons.org/licenses/by/4.0/), which permits unrestricted use, distribution, and reproduction in any medium, provided you give appropriate credit to the original author(s) and the source, provide a link to the Creative Commons license, and indicate if changes were made.

Funded by SCOAP ${ }^{3}$.

\section{Appendix A: Equations of motion for $S U(2)$ p-wave superconductors with hyperscaling violation}

In this appendix, we will construct the holographic p-wave superconductor models with hyperscaling violation by considering an $S U$ (2) Yang-Mills action [48]

$S=\int \mathrm{d}^{4} x \sqrt{-g}\left(-\frac{1}{4 \hat{g}^{2}} F_{\mu \nu}^{a} F^{a \mu \nu}\right)$,

where $\hat{g}$ is the Yang-Mills coupling constant and $F_{\mu \nu}^{a}=$ $\partial_{\mu} A_{\nu}^{a}-\partial_{\nu} A_{\mu}^{a}+\epsilon^{a b c} A_{\mu}^{b} A_{\nu}^{c}$ is the $S U$ (2) Yang-Mills field strength. The $A_{\mu}^{a}$ are the components of the mixed-valued gauge fields $A=A_{\mu}^{a} \tau^{a} \mathrm{~d} x^{\mu}$, where $\tau^{a}$ are the three generators of the $S U(2)$ algebra with commutation relation $\left[\tau^{a}, \tau^{b}\right]=\epsilon^{a b c} \tau^{c}$, and $\epsilon^{a b c}$ is the totally antisymmetric tensor with $\epsilon^{123}=+1$.

We will take the ansatz of the gauge fields as [48]

$A(u)=\phi(u) \tau^{3} \mathrm{~d} t+\psi(u) \tau^{1} \mathrm{~d} x$,

where we regard the $U(1)$ symmetry generated by $\tau^{3}$ as the $U(1)$ subgroup of $S U(2)$ and the gauge boson with nonzero component $\psi(u)$ along $x$-direction is charged under $A_{t}^{3}=\phi(u)$. From the gauge/gravity duality, $\phi(u)$ is dual to the chemical potential and $\psi(u)$ is dual to the $x$-component of some charged vector operator $\left\langle J_{x}\right\rangle$ on the boundary. The condensation of $\psi(u)$ will spontaneously break the $U(1)$ gauge symmetry and induce a phase transition, which can be interpreted as a p-wave superconductor phase transition in the boundary field theory.

From the Yang-Mills action (A1) and the solution (7) for $d=2, z=2$, we have the following equations of motion:

$\psi^{\prime \prime}+\left(\frac{f^{\prime}}{f}-\frac{1}{u}\right) \psi^{\prime}+\frac{r_{+}^{4} u^{2} \phi^{2}}{f^{2}} \psi=0$, $\phi^{\prime \prime}+\frac{1}{u} \phi^{\prime}-\frac{r_{+}^{2} \psi^{2}}{f} \phi=0$

where the prime denotes the derivative with respect to $u$.

\section{References}

1. J. Maldacena, Adv. Theor. Math. Phys. 2, 231 (1998)

2. J. Maldacena, Int. J. Theor. Phys. 38, 1113 (1999)

3. E. Witten, Adv. Theor. Math. Phys. 2, 253 (1998)

4. S.S. Gubser, I.R. Klebanov, A.M. Polyakov, Phys. Lett. B 428, 105 (1998)

5. S.A. Hartnoll, Class. Quant. Grav. 26, 224002 (2009)

6. C.P. Herzog, J. Phys. A 42, 343001 (2009)

7. G.T. Horowitz, Lect. Notes Phys. 828, 313 (2011). arXiv:1002.1722 [hep-th]

8. R.G. Cai, L. Li, L.F. Li, R.Q. Yang, Sci. China Phys. Mech. Astron. 58, 060401 (2015). arXiv:1502.00437 [hep-th]

9. S.S. Gubser, Phys. Rev. D 78, 065034 (2008)

10. S.A. Hartnoll, C.P. Herzog, G.T. Horowitz, Phys. Rev. Lett. 101, 031601 (2008)

11. S.A. Hartnoll, C.P. Herzog, G.T. Horowitz, J. High Energy Phys. 12, 015 (2008)

12. E. Brynjolfsson, U.H. Danielsson, L. Thorlacius, T. Zingg, J. Phys. A 43, 065401 (2010)

13. S.J. Sin, S.S. Xu, Y. Zhou, Int. J. Mod. Phys. A 26, 4617 (2011)

14. Y.Y. Bu, Phys. Rev. D 86, 046007 (2012)

15. R.G. Cai, H.Q. Zhang, Phys. Rev. D 81, 066003 (2010)

16. F.A. Schaposnik, G. Tallarita, Phys. Lett. B 720, 393 (2013)

17. E. Abdalla, J. Oliveira, A.B. Pavan, C.E. Pellicer. arXiv:1307.1460 [hep-th]

18. G. Tallarita, Phys. Rev. D 89, 106005 (2014). arXiv:1402.4691 [hep-th]

19. A. Lala, Phys. Lett. B 735, 396 (2014)

20. Z.X. Zhao, Q.Y. Pan, J.L. Jing, Phys. Lett. B 735, 438 (2014)

21. J.W. Lu, Y.B. Wu, P. Qian, Y.Y. Zhao, X. Zhang, Nucl. Phys. B 887, $112(2014)$

22. H. Guo, F.W. Shu, J.H. Chen, H. Li, and Z. Yu. arXiv: 1410.7020 [hep-th]

23. D. Momeni, R. Myrzakulov, L. Sebastiani, M.R. Setare, Int. J. Geom. Methods Mod. Phys. 12, 1550015 (2015)

24. K. Lin, E. Abdalla, A.Z. Wang, Int. J. Mod. Phys. D 24, 1550038 (2015)

25. A. Dector, Nucl. Phys. B 898, 132 (2015)

26. J.L. Jing, S.B. Chen, Q.Y. Pan, Phys. Lett. B 749, 376 (2015)

27. C. Charmousis, B. Gouteraux, B.S. Kim, E. Kiritsis, R. Meyer, J. High Energy Phys. 11, 151 (2010). arXiv:1005.4690 [hep-th]

28. B. Gouteraux, E. Kiritsis, J. High Energy Phys. 12, 036 (2011)

29. X. Dong, S. Harrison, S. Kachru, G. Torroba, H. Wang, J. High Energy Phys. 06, 041 (2012). arXiv:1201.1905 [hep-th]

30. M. Alishahiha, E.O. Colgain, H. Yavartanoo, J. High Energy Phys. 11, 137 (2012). arXiv:1209.3946 [hep-th]

31. M.A. Ganjali. arXiv:1508.05614 [hep-th]

32. L. Huijse, S. Sachdev, B. Swingle, Phys. Rev. B 85, 035121 (2012). arXiv:1112.0573 [cond-mat]

33. Z.Y. Fan, J. High Energy Phys. 08, 119 (2013)

34. Z.Y. Fan, Phys. Rev. D 88, 026018 (2013)

35. A. Lucas, S. Sachdev, K. Schalm, Phys. Rev. D 89, 066018 (2014)

36. L.K. Chen, H. Guo, F.W. Shu. arXiv:1511.01370 [hep-th]

37. S.J. Zhang, Q.Y. Pan, E. Abdalla. arXiv:1511.01841 [hep-th]

38. X.M. Kuang, J.P. Wu. arXiv:1511.03008 [hep-th]

39. D. Roychowdhury. arXiv:1511.06842 [hep-th]

40. S. Kachru, X. Liu, M. Mulligan, Phys. Rev. D 78, 106005 (2008). arXiv:0808.1725 [hep-th] 
41. X.M. Kuang, E. Papantonopoulos, B. Wang, J.P. Wu, J. High Energy Phys. 11, 086 (2014)

42. X.M. Kuang, E. Papantonopoulos, B. Wang, J.P. Wu, J. High Energy Phys. 04, 137 (2015)

43. Z.Y. Fan, J. High Energy Phys. 09, 048 (2013). arXiv:1305.2000 [hep-th]

44. G. Siopsis, J. Therrien, J. High Energy Phys. 05, 013 (2010)

45. G. Siopsis, J. Therrien, S. Musiri, Class. Quant. Grav. 29, 085007 (2012)

46. R.G. Cai, H.F. Li, H.Q. Zhang, Phys. Rev. D 83, 126007 (2011)

47. R.G. Cai, L. Li, H.Q. Zhang, Y.L. Zhang, Phys. Rev. D 84, 126008 (2011)

48. S.S. Gubser, S.S. Pufu, J. High Energy Phys. 11, 033 (2008). arXiv:0805.2960 [hep-th]

49. R.G. Cai, S. He, L. Li, L.F. Li, J. High Energy Phys. 12, 036 (2013). arXiv:1309.2098 [hep-th]

50. G.T. Horowitz, M.M. Roberts, Phys. Rev. D 78, 126008 (2008)

51. R.G. Cai, L. Li, L.F. Li, J. High Energy Phys. 01, 032 (2014). arXiv:1309.4877 [hep-th]

52. L.F. Li, R.G. Cai, L. Li, C. Shen, Nucl. Phys. B 894, 15 (2015). arXiv:1310.6239 [hep-th]

53. R.G. Cai, L. Li, L.F. Li, R.Q. Yang, J. High Energy Phys. 04, 016 (2014). arXiv:1401.3974 [gr-qc]

54. R.G. Cai, L. Li, L.F. Li, Y. Wu, J. High Energy Phys. 01, 045 (2014). arXiv:1311.7578 [hep-th]

55. Y.B. Wu, J.W. Lu, M.L. Liu, J.B. Lu, C.Y. Zhang, Z.Q. Yang, Phys. Rev. D 89, 106006 (2014). arXiv:1403.5649 [hep-th]

56. R.G. Cai, R.Q. Yang, Phys. Rev. D 91, 026001 (2015). arXiv: 1410.5080 [hep-th]

57. Y.B. Wu, J.W. Lu, Y.Y. Jin, J.B. Lu, X. Zhang, S.Y. Wu, C. Wang, Int. J. Mod. Phys. A 29, 1450094 (2014). arXiv:1405.2499 [hep-th]
58. L. Zhang, Q.Y. Pan, J.L. Jing, Phys. Lett. B 743, 104 (2015)

59. P. Chaturvedi, G. Sengupta, J. High Energy Phys. 04, 001 (2015)

60. Z.Y. Nie, R.G. Cai, X. Gao, L. Li, H. Zeng, Eur. Phys. J. C 75, 559 (2015). arXiv:1501.00004 [hep-th]

61. M. Rogatko, K.I. Wysokinski. arXiv:1508.02869 [hep-th]

62. I.M. Gelfand, S.V. Fomin, Calculaus of Variations, Revised English Edition, Translated and Edited by R.A. Silverman (Prentice-Hall, Inc. Englewood Cliffs, 1963)

63. H.F. Li, R.G. Cai, H.Q. Zhang, J. High Energy Phys. 04, 028 (2011)

64. H.B. Zeng, X. Gao, Y. Jiang, H.S. Zong, J. High Energy Phys. 05, $002(2011)$

65. J. Jing, Q. Pan, S. Chen, J. High Energy Phys. 11, 045 (2011)

66. S. Gangopadhyay, D. Roychowdhury, J. High Energy Phys. 05, 002 (2012)

67. S. Gangopadhyay, D. Roychowdhury, J. High Energy Phys. 05, 156 (2012)

68. S. Gangopadhyay, D. Roychowdhury, J. High Energy Phys. 08, 104 (2012)

69. Q.Y. Pan, J.L. Jing, B. Wang, S.B. Chen, J. High Energy Phys. 06, 087 (2012)

70. R. Banerjee, S. Gangopadhyay, D. Roychowdhury, A. Lala, Phys. Rev. D 87, 104001 (2013). arXiv: 1208.5902 [hep-th]

71. S. Gangopadhyay, Phys. Lett. B 724, 176 (2013)

72. X.M. Kuang, E. Papantonopoulos, G. Siopsis, B. Wang, Phys. Rev. D 88, 086008 (2013)

73. K. Lin, E. Abdalla, Eur. Phys. J. C 74, 3144 (2014)

74. L. Nakonieczny, M. Rogatko, Phys. Rev. D 90, 106004 (2014)

75. C.Y. Lai, Q.Y. Pan, J.L. Jing, Y.J. Wang, Phys. Lett. B 749, 437 (2015)

76. L. Nakonieczny, M. Rogatko, K.I. Wysokinski, Phys. Rev. D 92 , 066008 (2015) 